\title{
TRIM22: A Diverse and Dynamic Antiviral Protein
}

\author{
Clayton J. Hattlmann, Jenna N. Kelly, and Stephen D. Barr \\ Department of Microbiology and Immunology, Center for Human Immunology, The University of Western Ontario, \\ London, ON, Canada N6A 5C1
}

Correspondence should be addressed to Stephen D. Barr, stephen.barr@uwo.ca

Received 9 January 2012; Accepted 24 February 2012

Academic Editor: Abraham Brass

Copyright ( 12012 Clayton J. Hattlmann et al. This is an open access article distributed under the Creative Commons Attribution License, which permits unrestricted use, distribution, and reproduction in any medium, provided the original work is properly cited.

\begin{abstract}
The tripartite motif (TRIM) family of proteins is an evolutionarily ancient group of proteins with homologues identified in both invertebrate and vertebrate species. Human TRIM22 is one such protein that has a dynamic evolutionary history that includes gene expansion, gene loss, and strong signatures of positive selection. To date, TRIM22 has been shown to restrict the replication of a number of viruses, including encephalomyocarditis virus (EMCV), hepatitis B virus (HBV), and human immunodeficiency virus type 1 (HIV-1). In addition, TRIM22 has also been implicated in cellular differentiation and proliferation and may play a role in certain cancers and autoimmune diseases. This comprehensive paper summarizes our current understanding of TRIM22 structure and function.
\end{abstract}

\section{Introduction}

The TRIM gene family encodes a diverse group of proteins that are involved in many biological and antiviral processes. There are currently 100 known TRIM genes in the human genome and many of these genes are upregulated by multiple, distinct stimuli [1-3]. Historically, TRIM genes have been researched mainly for their antiviral properties; however this paradigm is changing. Two recent reports discussing the role of TRIM genes in autoimmunity and cancer highlight the importance of the TRIM family in the development of nonviral diseases $[4,5]$. Many TRIM genes also have a dynamic evolutionary history and the TRIM family has been shown to undergo extensive gene duplication in both primates and teleost fish $[1,6]$. In addition, several TRIM genes have experienced strong positive selection in primates [7]. Although the forces behind TRIM evolution remain unclear, it is possible that the TRIM family has evolved and continues to evolve, in response to new viral pathogens or endogenous danger signals. This paper provides an overview of the TRIM22 gene and summarizes its structure, evolution, expression, and antiviral activities.

\section{Structure}

TRIM proteins typically contain a conserved RBCC motif, which consists of an amino-terminal RING domain, one or two B-box domains, and a predicted coiled-coil region. Approximately $60 \%$ of TRIM proteins, including TRIM22, also contain a carboxyl-terminal domain B30.2 domain (Figure 1) [8, 9]. The RING domain of TRIM22 has homology with E3 ligases and has been shown to possess E3 ubiquitin ligase activity [9, 10]. The catalytic cysteine residues Cys 15 and Cys18 are essential for this activity and mediate the transfer of ubiquitin to target proteins (Figure 1) $[11,12]$. TRIM22 can also modify itself with ubiquitin which leads to proteasomal degradation $[10,11]$. Interestingly, the TRIM family represents one of the largest groups of E3 ubiquitin ligases and E3 ligase activity seems to be crucial for TRIM-mediated carcinogenesis [4]. In addition, E3 ligase activity is important for many TRIM-mediated antiviral activities and for TRIM22, it is required for the inhibition of EMCV, HBV, and HIV-1 [11, 13, 14].

TRIM proteins typically contain one or two B-box domains, although B-box 1 is never present without B-box 2 , and the two domains have different consensus sequences 

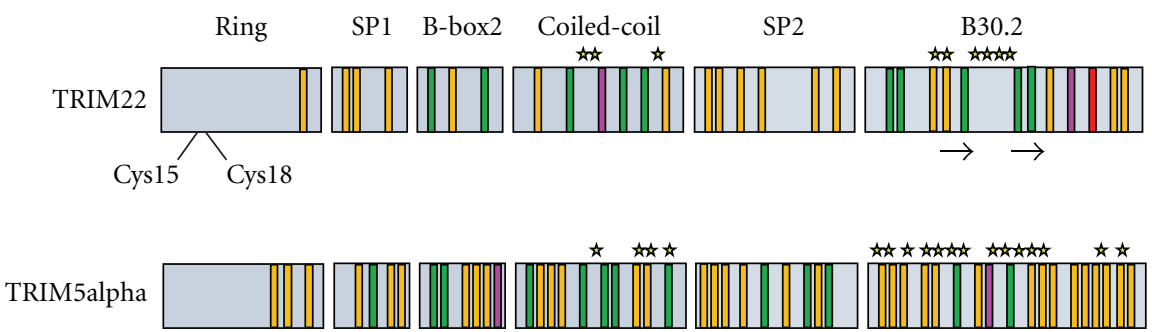

FIGURE 1: Structure and variability of TRIM22 and TRIM5 $\alpha$ protein domains. TRIM22 contains an amino-terminal RING domain, one B-box domain (B-box 2), a coiled-coil region, and a carboxyl-terminal B30.2 domain (SP1 = Spacer 1 and SP2 = Spacer 2). Two cysteine residues (Cys15 and Cys18) in the RING domain are required for the E3 ligase activity of TRIM22, and a number of positively selected amino acids are found in the coiled-coil and B30.2 domains. The location and spacing of positively selected amino acids in TRIM22 are similar to those found in TRIM $5 \alpha$, which may reflect species-specific pathogenic pressures. The approximate location of positively selected amino acids in TRIM22 and TRIM5 $\alpha$ is denoted with a star, and the location of the $\beta 2-\beta 3$ surface loop of TRIM 22 is also indicated (arrows). Single nucleotide polymorphisms (SNPs) in the coding regions of TRIM22 and TRIM5 $\alpha$ are shown as vertical bars, along with the type of mutation that each SNP can generate (green: nonsynonymous mutations; yellow: missense mutations; pink: frameshift mutations; red: nonsense mutations).

$[15,16]$. TRIM22 contains one B-box domain (B-box 2), of which no clear function has been assigned (Figure 1). Certain B-box 2 mutations have been shown to affect viral recognition by other TRIM proteins, such as TRIM $5 \alpha$. Similar to TRIM22, TRIM5 $\alpha$ has been shown to inhibit HIV-1 replication albeit at an earlier stage in the viral lifecycle. Interestingly, the human orthologue of TRIM $5 \alpha$ only modestly inhibits HIV-1 replication whereas the rhesus orthologue of TRIM $5 \alpha$ (rhTRIM5 $\alpha$ ) has potent anti-HIV-1 activity [17]. Several mechanisms of rhTRIM $5 \alpha$-mediated HIV-1 inhibition have been proposed; however, the favoured mechanism involves rhTRIM $5 \alpha$ binding to the HIV-1 core and disruption of the normal uncoating process (reviewed in $[18,19])$. For rhTRIM $5 \alpha$, the RING and B-box 2 domains promote its dimerization and higher-order self-association on the HIV-1 capsid [17]. It is unknown whether the B-box 2 domain of TRIM22 is required for higher-order self-association; however, it has been shown to play a role in the nuclear localization of TRIM22 [20].

The coiled-coil domain contains multiple predicted hypersecondary structures and intertwined $\alpha$-helices [21]. In TRIM proteins, the coiled-coil domain is thought to promote homo-oligomerization, as its deletion prevents TRIM protein self-association [22]. Homo-oligomerization can be important for the formation of higher-molecular-weight complexes that define specific subcellular structures, such as nuclear bodies $[21,22]$. Although the role of the coiledcoil region of TRIM22 remains unclear, self-association is a function of the coiled-coil region in other TRIM proteins. For example, the coiled-coil region of rhTRIM $5 \alpha$ is required for $\operatorname{rhTRIM} 5 \alpha$ trimerization and may be involved in the formation of cytoplasmic bodies. Importantly, rhTRIM $5 \alpha$ trimerization is thought to drive its interaction with the HIV1 capsid and the coiled-coil region is required for rhTRIM $5 \alpha-$ mediated HIV-1 restriction [17, 23]. TRIM22 has also been shown to form trimers and to restrict HIV-1 replication but it is unknown whether the coiled-coil domain is required for these processes [14, 22, 24-26].

The B30.2 domain of TRIM proteins consists of two separate domains called the PRY and SPRY domains that form a putative protein-protein interaction site [27, 28]. This interaction site is likely important for the antiviral activities of TRIM22 and other TRIM proteins. Indeed, the B30.2 domain of rhTRIM $5 \alpha$ is required for trimerization and HIV-1 restriction [23]. Three hyper-variable regions in the B30.2 domain of rhTRIM5 $\alpha$ are thought to form the binding surface for the HIV-1 capsid protein [29]. In addition, these hypervariable regions confer the virus specificity of rhTRIM $5 \alpha$. The B30.2 domain of TRIM 22 also contains these three hypervariable regions but their role in HIV-1 restriction has not yet been established. Similar to $\operatorname{rhTRIM} 5 \alpha$, the hypervariable regions in TRIM22 are highly polymorphic and contain a large number of positively selected amino acids (Figure 1) [7]. It will be interesting to learn if the B30.2 domain of TRIM22 confers specificity for targets such as viral pathogens. Notably, the B30.2 domain is required for the formation of nuclear bodies $[13,20,30]$.

\section{Evolution of TRIM22}

The human TRIM22 gene is located on chromosome 11, immediately adjacent to the TRIM5, TRIM6, and TRIM34 genes $[7,31]$. The origins of TRIM22, and the entire TRIM5/6/22/34 gene cluster, can be traced back to the Cretaceous period, sometime after the divergence of Metatherian (marsupial) and Eutherian (placental) mammals (Figure 2). Previous studies have shown that the TRIM5/6/22/34 locus is absent in Metatherian mammals such as opossum and chicken but presents in the major Eutherian groups containing cow, dog, and human [7]. Thus, this gene cluster must have emerged after the Metatherian-Eutherian division but before the separation of the major Eutherian groups. Taken together, this dates the birth of TRIM22 (along with TRIM5, TRIM6 and TRIM34) to approximately 90-180 million years ago (Figure 2) [7].

The TRIM5/6/22/34 gene cluster likely arose through tandem gene duplication, as these four TRIM genes are close human paralogs and because major gene rearrangements have been documented in this chromosomal region 


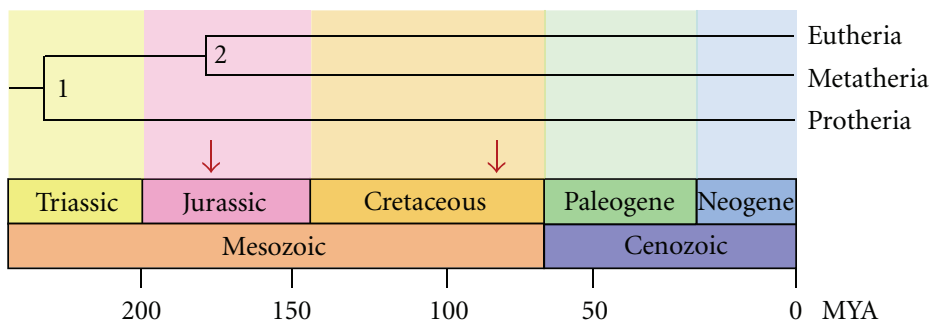

Figure 2: Timeline of Metatherian and Eutherian mammalian evolution showing the emergence of TRIM22. The divergence of Metatherian (marsupial) and Eutherian (placental) mammals occurred approximately 180 million years ago in the Jurassic period of the Mesozoic era. The TRIM22 gene emerged sometime after this division, as it is absent in Metatherian mammals but present in all major Eutherian groups. In addition, since TRIM22 is present in all Eutherian mammals, it must have emerged before further Eutherian division occurred (approximately 90 million years ago). Taken together, this dates the birth of TRIM22 to approximately 90-180 million years ago. The predicted window of time for TRIM22 emergence in Eutherian mammals is demarcated with two red arrows. MYA: millions of years.

$[1,7,32]$. Gene duplication plays a major role in evolution and TRIM genes have been shown to undergo extensive gene duplication in both primates and teleost fish $[1,6]$. One of the most important outcomes of gene duplication is neofunctionalization, whereby one copy of the duplicate gene acquires a novel, beneficial function, and the other copy of the gene retains its original function [33-35]. This type of gene manipulation is a potent driver of evolution because it allows an organism to create new, potentially advantageous genes without disrupting the integrity of the original gene.

Recently, a genomic analysis of a different branch of the TRIM gene family identified several TRIM genes on chromosome 11 that have given rise to multiple TRIM paralogs in humans and African apes [1]. A group of 7 TRIM genes that are present in all Eutherian mammals (TRIM43, TRIM48, TRIM49, TRIM51, TRIM53, TRIM64, and TRIM77) were shown to spawn 11 new TRIM genes in certain primates and 6 new TRIM genes in humans, primarily through segmental duplications [1]. These new TRIM genes have presumably evolved and adapted to react against more recently emerged pathogenic threats. In addition, a Han Chinese woman with 12 new TRIM genes was identified, documenting for the first time TRIM gene copy number variation in humans [1]. Given its role in antiviral immunity, TRIM22 probably emerged in a similar manner as a means of counteracting new viral pathogens; however the exact selective pressures giving rise to the TRIM22 gene remain unclear.

According to a recent study, TRIM genes can be divided into two main groups based on their structural similarities and evolutionary properties [36]. Group 1 members have two B-box domains, have variable C-terminal domains, and are represented in both vertebrate and invertebrate species. In contrast, Group 2 members have only one B-box domain (B-box 2), are characterized by a C-terminal SPRY domain, and are found only in vertebrates. In addition, Group 2 genes are younger and smaller and evolve more rapidly than Group 1 genes [36]. Compared to some other TRIM genes, TRIM22 is young and has evolved under strong positive selection, thus TRIM22 (along with the TRIM5/6/22/34 gene cluster) is classified as a Group 2 gene. Interestingly, the authors suggest that Group 2 genes may act as TRIM gene reservoirs, spawning new genes to respond to species-specific changes at the host-pathogen interface. Consistent with this interpretation, there are a number of positively selected amino acids in TRIM22 which all cluster at predicted virus interaction sites in the coiled-coil and B30.2 domains (Figure 1) $[7,36]$.

Within the TRIM5/6/22/34 gene cluster, TRIM22 and TRIM5 have a unique evolutionary relationship. In some Eutherian groups, such as cow, there are multiple copies of the TRIM5 gene and no TRIM22 gene. However in others such as dog, the TRIM22 gene is present and the TRIM5 gene is absent [7]. In addition, the strong positive selection that each of these two genes has experienced over millions of years has occurred in a mutually exclusive manner. This type of anticorrelative pattern is probably due to genetic linkage between the two genes, whereby positive selection of an advantageous mutation in one gene indirectly leads to the selection of a linked mutation in the other [7]. The location and spacing of positively selected amino acids in TRIM22 is very similar to those found in TRIM5 $\alpha$ (Figure 1). In both proteins, the positively selected amino acids are located in the coiled-coil and B30.2 domains, which is interesting because their amino acid sequences are actually the least similar in these regions. The majority of positively selected amino acids in TRIM22 are found within the $\beta 2-\beta 3$ surface loop of the B30.2 domain, an area that is important for $\mathrm{HIV}-1$ recognition in TRIM5 $\alpha$ (Figure 1) [7, 37, 38]. It is possible that TRIM22 and TRIM5 $\alpha$ once possessed a similar antiretroviral mechanism, and that they evolved over time to respond to species-specific pathogenic pressures. Indeed, many studies have shown that rhesus TRIM5 $\alpha$, but not human TRIM5 $\alpha$, can potently inhibit HIV-1 replication $[18,39]$. In contrast, human TRIM22 can inhibit HIV-1 replication and thus may have evolved to compensate for the loss of TRIM $5 \alpha$ 's anti-HIV function.

The TRIM22 gene has a dynamic evolutionary history that includes gene expansion, gene loss, and strong signatures of positive selection in primates $[1,6,7,36]$. The high number of nonsynonymous mutations found in TRIM22, along with its classification as a Group 2 TRIM gene, suggests that this gene continues to evolve at a rapid pace. Given the volatile state of other TRIM genes in chromosome 11 , it is possible that the TRIM5/6/22/34 gene cluster takes part in 
TABLE 1: Summary of the localization patterns observed for TRIM22.

\begin{tabular}{|c|c|c|c|c|}
\hline Localization & Pattern & Cell Type & Epitope Tag & Reference \\
\hline \multirow{7}{*}{ Cytoplasm } & Diffuse & $293 \mathrm{~T}$ & GFP or V5/His & {$[40]$} \\
\hline & Diffuse & COS7 & GFP or V5/His & {$[40]$} \\
\hline & Diffuse & $\mathrm{HeLa}$ & Endogenous & {$[40]$} \\
\hline & Diffuse with speckles/bodies & $\mathrm{HeLa}$ & GFP & {$[22]$} \\
\hline & Diffuse & $\mathrm{HeLa}$ & GFP or V5/His & {$[40]$} \\
\hline & Diffuse & PBMCs & Endogenous & {$[40]$} \\
\hline & Diffuse with speckles/bodies & U2OS & GFP & {$[22]$} \\
\hline \multirow{8}{*}{ Cytoplasm \& Nucleus } & Nucleoplasmic, with nuclear bodies ${ }^{1}$ & $\mathrm{ABC} 28$ & Endogenous & {$[30]$} \\
\hline & Diffuse throughout, or nuclear bodies ${ }^{2}$ & HeLa & EGFP & {$[30]$} \\
\hline & Nucleoplasmic, with nuclear bodies & HeLa & Endogenous & {$[30]$} \\
\hline & Diffuse, with cytoplasmic bodies ${ }^{3}$ & $\mathrm{HeLa}$ & FLAG & {$[43]$} \\
\hline & Nucleoplasmic with $\mathrm{NB}^{4}$ & MCF7 & EGFP, EYFP, or FLAG & {$[30]$} \\
\hline & Nucleoplasmic, with nuclear bodies & MCF7 & Endogenous & {$[30]$} \\
\hline & Nucleoplasmic and cytoplasmic & T47D & Endogenous & {$[30]$} \\
\hline & Diffuse with speckles ${ }^{5-7}$ & $\mathrm{U} 2 \mathrm{OS}$ & Endogenous & {$[42]$} \\
\hline \multirow{5}{*}{ Nucleus } & Aggregates/bodies & 293 & Myc & {$[41]$} \\
\hline & Aggregates/bodies & COS7 & Myc & {$[10]$} \\
\hline & Diffuse with speckles/bodies & HepG2 & Endogenous & [13] \\
\hline & Diffuse with speckles/bodies & HepG2 & Myc & {$[13]$} \\
\hline & Diffuse with bodies & MCF7 & FLAG & {$[20]$} \\
\hline
\end{tabular}

${ }^{1}$ Some colocalization with fibrillarin (Nucleoli).

${ }^{2}$ Pattern changes with cell cycle phase: (G0/G1: nuclear bodies; S-phase: nuclear speckles and cytoplasmic; mitosis: diffuse throughout cell).

${ }^{3}$ TRIM22 plasmid was coexpressed with Rhesus TRIM5 $\alpha$.

${ }^{4}$ Partial colocalization with Cajal bodies.

${ }^{5}$ Potential colocalization with calnexin (Endoplasmic reticulum).

${ }^{6}$ Localization was primarily cytoplasmic when cells were fixed with paraformaldehyde, or both cytoplasmic and nuclear when fixed with ice-cold methanol.

${ }^{7}$ Partial colocalization with the centrosome.

gene and/or segmental duplication in humans. Presumably, individuals with an increased number of these TRIM genes may have an augmented antiviral response and could be particularly adept at controlling retroviral infections. Similar to copy number variations, a number of single nucleotide polymorphisms (SNPs) exist in TRIM22 that may influence its antiviral capacity or biological function for that matter. For instance, there are two documented frameshift mutations and one documented nonsense mutation in the National Center for Biotechnology Information SNP database for the TRIM22 gene (Figure 1). If present, these SNPs would generate different truncated versions of the TRIM22 protein, which may alter its structure, E3 ubiquitin ligase activity and/or antiviral function. There are also twenty documented missense mutations in the TRIM22 gene, the majority of which are found in its B30.2 domain (Figure 1). Many of these SNPs have the potential to impact TRIM22 function and their presence or absence may contribute to individual differences in TRIM22-mediated activities.

\section{Biological Functions of TRIM22}

4.1. TRIM22 Localization. There are several contradictory reports detailing the subcellular localization of TRIM22. Some reports have observed that TRIM22 localizes predominantly to the cytoplasm $[22,40]$ or to the nucleus $[10,13,20,41]$, whereas other reports have observed that TRIM22 can localize to both the cytoplasm and the nucleus (Table 1) [30, 42, 43]. The pattern of localization also varied between diffuse, speckled, and aggregated. A number of explanations have been given in the literature for the differences in localization, including whether the expression was endogenous (e.g., IFN-treatment) or exogenous (e.g., overexpression). In addition, the method of fixation and the type of epitope tag used for detection have also been reported to affect the localization pattern. Given the diverse range of cell lines used in these studies, it is also possible that cell type-specific factors influence the localization of TRIM22.

A number of determinants affecting TRIM22 localization have been identified. A bipartite nuclear localization signal (NLS) located in the Spacer 2 domain of TRIM22 was shown to be necessary, but not sufficient, for nuclear localization [20]. Although there are no known NLSs present in the B30.2/SPRY domain, several groups have shown that this domain is required for nuclear localization [13, 20, 40, 41]. More specifically, Val 493 and Cys 494 of the B30.2 domain were shown to be critical for nuclear localization and the formation of nuclear bodies [20]. In an independent study, amino acids Ser 395, Lys 396, and Ser 400 located in variable loops 1 and 3 of the B30.2 domain were shown to be important for certain localization patterns of TRIM22 [40].

In some cell types, TRIM22 localizes in the nucleus as punctate bodies, which have been shown to partially colocalize with Cajal bodies [20]. Cajal bodies play important roles in RNA processing and modification as well as in cell cycle progression [44]. TRIM22 also interacts with p80coilin, which is a major component of Cajal bodies. Similar to Cajal bodies, TRIM22 localization has been shown to change during the cell cycle. In G0/G1 TRIM22 localizes 
in nuclear bodies, in S-phase it localizes in a more diffuse and speckled pattern throughout the nucleus, and during mitosis it assumes a diffuse pattern in both the nucleus and cytoplasm [30]. In an independent study, TRIM22 was shown to colocalize with the centrosome independently of the cell cycle and also with vimentin-containing aggresomelike structures next to the endoplasmic reticulum [42]. From these data, it appears that multiple factors influence the localization of TRIM22, possibly indicating that TRIM22 has several biological roles.

4.2. Antiviral Function of TRIM22. Several reports including published transcriptional profiling datasets (e.g., GDS1096, GDS3113, and GDS596) deposited in the Gene Expression Omnibus database repository (http://www.ncbi.nlm.nih. gov/gds) show that TRIM22 is ubiquitously expressed in several human tissues and is highly upregulated in response to Type I and II interferons (Table 2) [7, 13, 14, 24, 25, 4550]. Interestingly, the $5^{\prime}$ flanking region of the TRIM 22 gene contains two regions matching the consensus sequence for an IFN-stimulating response element (ISRE) and a third region matching that for an IFN- $\gamma$ activation site (GAS); however ISRE1 or GAS is not required for IFN$\gamma$ induction of TRIM22. In contrast, the ISRE2 plus six upstream nucleotides (extended ISRE) is capable of binding IFN regulatory factor 1 (IRF1) in a manner dependent on the chromatin remodelling enzyme Brahma-related gene 1 (BRG1) $[48,49]$. Furthermore, this extended ISRE appears to be important for both stimulation by IFN- $\alpha$ and IFN- $\gamma$ as well as for basal TRIM22 expression [48]. The significant upregulation of TRIM22 in response to IFNs, together with the finding that TRIM22 has evolved under strong positive selection for millions of years, suggests that TRIM22 plays an important fundamental role in cell biology. To date, several lines of evidence suggest that this role is as an antiviral factor.

Human TRIM22 was first discovered by Tissot and Mechti in 1995 during a search for IFN-induced genes in Daudi cells, where exogenous expression of TRIM22 was shown to downregulate transcription from the HIV-1 LTR [45]. Although this was performed using a luciferase reporter gene under the transcriptional control of the HIV-1 LTR and not in the context of the entire HIV-1 proviral genome, it provided the first evidence suggesting that TRIM22 blocks HIV-1 transcription and replication. In 2006, Bouazzaoui et al. showed that TRIM22 was highly upregulated in primary monocyte-derived macrophages (MDMs) in response to HIV-1 infection, IFN $\alpha$ treatment, or stimulation with lipopolysaccharide (LPS). They provided the first evidence that TRIM22 can restrict HIV-1 replication in vitro by showing that exogenous expression of TRIM22 inhibited HIV-1 infection by $50-90 \%$ in $293 \mathrm{~T}$ cells modified to express the CD4 and CCR5 receptors and in primary MDM. Furthermore, cotransfection of TRIM22 with a threeplasmid system for replication-defective HIV-1 resulted in reduced infectious titres of pseudotyped virus, suggesting that TRIM22 inhibited a late stage of HIV-1 pseudoparticle production and/or subsequent infection with the pseudotyped virus [24].
In 2008, Barr et al. showed that TRIM22 was an integral part of the Type I interferon-induced inhibition of HIV-1 replication and provided the first mechanistic data for the inhibition of HIV-1 replication by TRIM22. TRIM22 expression in several human cell lines potently inhibited HIV-1 replication, and interestingly, analysis of Gag production in those cells revealed that TRIM22 may inhibit HIV-1 replication by two different mechanisms. In the HOS and HeLa cell lines, TRIM22 inhibited HIV-1 particle production by interfering with the trafficking of the Gag polyprotein to the plasma membrane. Since TRIM22 and Gag proteins interact, and that the E3 ligase activity of TRIM22 is required for this restriction [14], it is possible that TRIM22 posttranslationally modifies Gag, resulting in altered Gag trafficking to the plasma membrane. In the U2OS and 143B cell lines, TRIM22 inhibited HIV-1 particle production by inhibiting the accumulation of intracellular Gag protein [14]. Although no mechanism of restriction was identified in U2OS or 143B cells, several possibilities could explain the decrease in intracellular Gag protein levels, including inhibition of transcription from the LTR as previously suggested $[25,45]$, or degradation of the Gag RNA and/or polyprotein. Given that TRIM22 exhibits cell typespecific differences in localization (as discussed earlier), it is likely that the mechanism of TRIM22-induced restriction of HIV-1 particle production is cell type-specific and/or dependent on the subcellular localization of TRIM22. Future experiments are required to further elucidate the mechanism of TRIM22-induced inhibition of HIV-1 particle production (Figure 3).

TRIM22 was also independently identified and shown to inhibit HIV-1 replication by several laboratories [25]. Following observations made by Franzoso et al. in 1994 that clones of the U937 promonocytic cell line were either permissive or nonpermissive to HIV-1 replication, Kajaste-Rudnitski et al. (2011) identified TRIM22 as the only known restriction factor that was expressed in the nonpermissive and absent from the permissive U937 cells. Using a luciferase reporter plasmid under the control of the HIV-1 LTR, they showed that LTR-mediated transcription was decreased 7-10-fold in nonpermissive clones. They also showed that by knocking down TRIM22 expression in nonpermissive cells, the levels of transcription from the LTR approached those observed in permissive cells. Exogenous expression of TRIM22 in permissive clones also decreased LTR transcription to levels comparable to those observed in nonpermissive clones. Further investigation revealed that TRIM22 inhibited basal and phorbol myristate acetateionomycin-induced HIV-1 transcription. These effects were independent of $\mathrm{NF} \kappa \mathrm{B}, \mathrm{HIV}-1$ Tat and the $\mathrm{E} 3$ ubiquitin ligase activity of TRIM22 [25]. It is important to note that all direct evidence showing that TRIM22 inhibits HIV-1 transcription has been through the use of LTR-driven reporter constructs. It will be important to test the effects of TRIM22 on HIV-1 LTR transcription in the context of full-length replicationcompetent HIV-1.

In 2011, Singh et al. provided the first clinically relevant evidence supporting a role for TRIM22 as an antiHIV-1 effector in vivo. They showed that expression of 


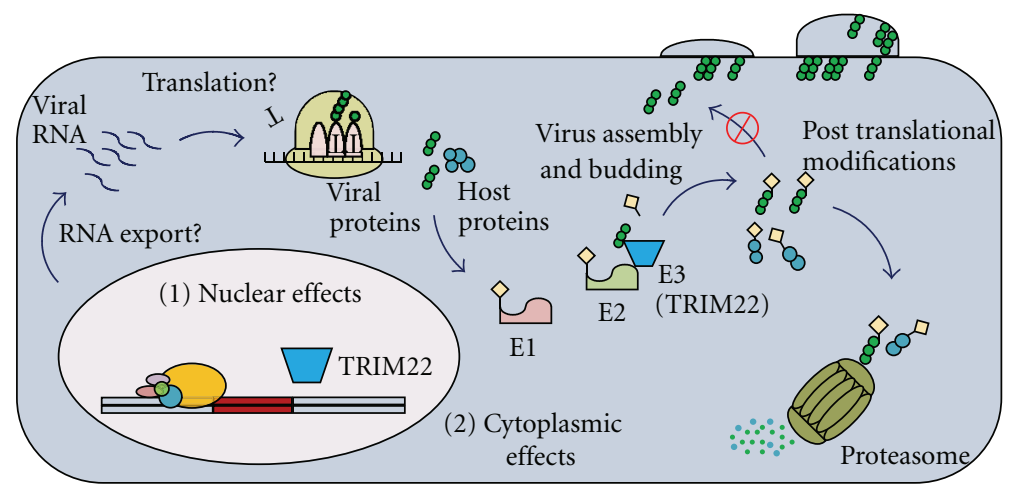

FIgure 3: Possible mechanisms of TRIM22 antiviral functions. Based on current reports, TRIM22 can inhibit viral replication through nuclear-associated effects such as inhibiting viral transcription. Although not investigated to date, RNA export and translation are also potential targets of TRIM22. Given its E3 ligase activity, TRIM22 may posttranslationally modify host or viral proteins that are required for viral assembly and/or budding. Posttranslational modifications occur when an E1 activating enzyme (E1), E2 conjugating enzyme (E2), and E3 ligase protein (E3) work together to transfer ubiquitin or ubiquitin-like molecules to a target protein. These modifications could target the protein for proteasomal degradation or alter its subcellular localization or ability to interact with other proteins or DNA.

TRIM22 in peripheral blood mononuclear cells (PBMCs) of HIV-1-infected individuals was significantly increased in patients after HIV-1 infection. Importantly, infected patients expressing higher TRIM22 levels exhibited significantly lower viral loads and significantly higher CD4+ T-cell counts [26]. These findings are quite significant, as this suggests that TRIM22 has a potential effect on the severity and/or progression of HIV-1 infection. Additional research on the role of TRIM22 during primary infection will be important to provide a greater understanding of the effects TRIM22 may have on HIV-1 replication in vivo.

The antiviral activities of TRIM22 are not limited to HIV-1. In 2009, Eldin et al. identified TRIM22 as a potent inhibitor of encephalomyocarditis virus (EMCV) replication. TRIM22 was shown to interact with the EMCV 3C protease via the C-terminal domain of TRIM22, and expression of TRIM22 corresponded with increased ubiquitination of the 3C protease (Figure 3). 3C protease is essential for successful viral replication and has several roles, including processing of the viral polyprotein and inhibition of the host immune defences [11]. There are also reports that TRIM22 may play an important role in protecting the liver from viral pathogens. In 2009, Gao et al. reported that TRIM22 is highly upregulated in response to type I or II IFN in the hepatocellular carcinoma cell line HepG2. Cotransfection of plasmids encoding TRIM22 and replication-competent hepatitis B virus (HBV) inhibited the accumulation of HBV antigens in the supernatants of cells and significantly reduced levels of intracellular HBV RNA and DNA replication intermediates. Similar results were observed in the sera of mice during codelivery of plasmids to mouse livers, showing that TRIM22 can restrict HBV infection in an in vivo system. Using a luciferase reporter plasmid, they showed that TRIM22 downregulates expression from the HBV core promoter (Figure 3). This mechanism of action was dependent on the nuclear localization of TRIM22 and its E3 ubiquitin ligase activity [13]. Although there is no direct evidence for a protective role of TRIM22 against $\mathrm{HBV}$ in primates, TRIM22 expression is significantly upregulated during clearance of HBV in chimpanzees [51]. Moreover, TRIM22 expression is significantly upregulated during clearance of hepatitis $\mathrm{C}$ virus (HCV) in chimpanzees [52]. These findings are paralleled in human infections, as TRIM22 is significantly upregulated in cirrhotic liver from HCV patients and patients with mild chronic HCV infection and no fibrosis [53]. Further research is needed to assess the role of TRIM22 in inhibiting HBV and HCV in vivo.

In further support of the notion that TRIM22 is involved in the host antiviral response, TRIM22 expression is modulated in response to several other viruses and viral antigens (Table 2). TRIM22 expression is upregulated in response to infection with rubella virus [54] and EpsteinBarr virus (EBV) [55] and downregulated during infection with human papillomavirus type 31 [56]. A couple intriguing reports elude to the possibility that TRIM22 may also contribute to viral latency. Exogenous expression of TRIM22 significantly upregulates expression of the EBV latent membrane protein 1 (LMP-1) [55]. LMP-1 is required for latency during EBV infection and appears to induce an antiviral state by upregulating expression of several ISGs via an IFN- and STAT1-independent mechanism. The Kaposi's sarcoma-associated herpesvirus (KSHV) latency-associated nuclear antigen (LANA) also activates several ISGs including TRIM22, which was shown to be upregulated by LANA both in culture and in tissues from KSHV lesions. LANA also repressed transcription from the HIV-1 LTR, an NF $\kappa$ B consensus sequence, and the SV40 promoter [57]. Furthermore, TRIM22 is expressed in resting $\mathrm{T}$ cells, which are known reservoirs of latent HIV-1, and is strongly repressed during Tcell activation [47]. Although much more research is needed to directly implicate TRIM22 in viral latency, it is tempting to hypothesize that TRIM22 contributes to viral latency.

4.3. Other Functions of TRIM22. Several reports in the literature suggest that TRIM22 may have a role in other biological processes, such as cell differentiation and proliferation. One 
TABLE 2: Summary of factors that alter TRIM22 expression.

\begin{tabular}{|c|c|c|c|}
\hline Stimulation & Change & Tissue & Reference \\
\hline \multicolumn{4}{|l|}{ Cytokines } \\
\hline IFN- $\alpha$ & increase & CEM, Jurkat, and THP-1 cells & {$[26]$} \\
\hline IFN- $\alpha$ & increase & H9 cells & {$[47]$} \\
\hline IFN- $\alpha$ & increase & HepG2 cells & {$[13]$} \\
\hline IFN- $\alpha$ & increase & Primary MDM & {$[24]$} \\
\hline IFN- $\alpha$ & increase & U937 & {$[25]$} \\
\hline IFN- $\alpha$ & increase & U-937-4 cells & {$[46]$} \\
\hline IFN- $\alpha / \beta$ & increase & Daudi, and HeLa cells & {$[45]$} \\
\hline IFN- $\beta$ & increase & HOS cells & {$[14]$} \\
\hline IFN- $\gamma$ & increase & HeLa cells & {$[30,45]$} \\
\hline IFN- $\gamma$ & increase & HepG2 cells & {$[13,48,49]$} \\
\hline IFN- $\gamma$ & increase & MCF7 cells & {$[30]$} \\
\hline IL-1- $\beta$ & increase & Coronary artery endothelium & {$[58]$} \\
\hline IL-2 & increase & $\mathrm{CD} 4+, \mathrm{CD} 8+, \mathrm{NK}$ cells & {$[50]$} \\
\hline IL-15 & increase & $\mathrm{CD} 4+, \mathrm{CD} 8+, \mathrm{NK}$ cells & {$[50]$} \\
\hline Progesterone & increase & $\mathrm{ABC} 28$, and T47D cells & {$[30]$} \\
\hline TNF- $\alpha$ & increase & Coronary artery endothelium & {$[58]$} \\
\hline \multicolumn{4}{|l|}{ Antigens/Infections } \\
\hline EBV infection ${ }^{1}$ & increase & BL41-EBV cells ${ }^{1}$ & {$[55]$} \\
\hline EBV LMP-1 & increase & DG75 cells & {$[55]$} \\
\hline Hepatitis B virus infection ${ }^{2}$ & increase & Liver tissue ${ }^{2}$ & {$[51]$} \\
\hline Hepatitis $\mathrm{C}$ virus infection ${ }^{2}$ & increase & Liver tissue $^{2}$ & {$[52]$} \\
\hline Hepatitis $\mathrm{C}$ virus infection & increase & Liver tissue & {$[53]$} \\
\hline HIV-1 infection & increase & Immature DC & {$[55]$} \\
\hline HIV-1 infection & increase & Primary MDM & {$[24]$} \\
\hline HIV-1 infection & increase & Primary PBMCs & {$[26]$} \\
\hline HIV-1 Tat & increase & Immature DC & {$[55]$} \\
\hline HPV infection & decrease & Human keratinocytes & {$[56]$} \\
\hline KSHV infection & increase & KSHV lesion & {$[57]$} \\
\hline KSHV LANA & increase & BJAB cells & {$[57]$} \\
\hline LPS & increase & Primary MDM & {$[24]$} \\
\hline Rubella virus infection & increase & ECV304 cells & {$[54]$} \\
\hline \multicolumn{4}{|l|}{ Activation/Differentiation/Cell Cycle } \\
\hline $1 \alpha, 25$-dihydroxyvitamine $\mathrm{D} 3^{3}$ & increase & Primary MDM & {$[24]$} \\
\hline Anti-CD2 & increase & Primary T cells & {$[47]$} \\
\hline Anti-CD2/CD28 & decrease & Primary T cells & {$[47]$} \\
\hline Anti-CD2/CD28/CD3 & decrease & $\mathrm{CD} 4+, \mathrm{CD} 8+, \mathrm{NK}$ cells ${ }^{4}$ & {$[50]$} \\
\hline All-trans retinoic acid & increase & HL60 and NB4 cells & {$[46]$} \\
\hline All-trans retinoic acid & increase & Primary MDM & {$[24]$} \\
\hline p53 & increase & K562 and U-937-4 cells ${ }^{5}$ & {$[46]$} \\
\hline p73 & increase & U-937-4 cells & {$[46]$} \\
\hline Pioglitazone & increase & Primary MDM & [24] \\
\hline UV-irradiation $^{6}$ & increase & MCF-7 cells & {$[46]$} \\
\hline \multicolumn{4}{|l|}{ Disease } \\
\hline SLE & decrease & CD4+ T cells from SLE patient & {$[59]$} \\
\hline
\end{tabular}


TABle 2: Continued.

\begin{tabular}{lcc}
\hline Stimulation & Change & Tissue \\
\hline \multicolumn{1}{c}{ Wilms tumor } & decrease & Tumor tissue \\
${ }^{1}$ BL41 cells that are latently infected with EBV. & & Reference \\
${ }^{2}$ From infected chimpanzees. \\
${ }^{3}$ Hormonally active form of Vitamin D. \\
${ }^{4}$ Only reached significance in CD8+ cells. \\
${ }^{5}$ Cells lack endogenous p53 but stably express a plasmid encoding p53 under control of a temperature-sensitive promoter. Cells were grown at the permissive \\
temperature $\left(32^{\circ} \mathrm{C}\right)$ to induce p53 expression.
\end{tabular}

group showed that the expression of TRIM22 is directly activated by $\mathrm{p} 53$ in myeloid cells via a functional p53response element in intron 1 of the TRIM22 gene [46]. They also showed that the p53-family member p73 can bind to this response element and activate TRIM22 expression [46]. Since p73 has been linked to the differentiation of leukemic cells [62], the authors speculated that TRIM22 may be involved in cell differentiation. Another group reported that TRIM22 expression is significantly upregulated during differentiation of the promyelocytic cell line NB4 [63]. They also showed that TRIM22 expression is high in monocytes and early granulocytes but decreases in the lymphocyte and late granulocyte populations and is undetectable in erythroid cells [63]. Obad et al. (2004) provided the first direct evidence supporting an antiproliferative role for TRIM22 by showing that overexpression of TRIM22 in the promonocytic cell line U937 resulted in decreased clonogenic growth [46]. An inverse correlation between TRIM22 expression and cell differentiation has also been reported, showing that TRIM22 is highly expressed in human immature $\mathrm{CD} 34^{+}$bone marrow progenitor cells, but declines in mature populations [63]. Despite the correlations of TRIM22 expression levels with cell differentiation and proliferation, the evidence lacks key experiments such as loss-of-function studies (i.e., TRIM22 knockdown) to conclusively implicate TRIM22 as a key player in any of these processes.

A couple of reports have associated TRIM22 with human disease. Downregulation of TRIM22 expression is associated with progression, relapse and increased mortality in cases of Wilms tumor $[60,61]$. Although TRIM22 is a p53responsive gene and may promote cell-cycle arrest [46], its role in tumour development and progression, including Wilms tumor, is yet to be determined. The involvement of TRIM proteins in cancer is not unprecedented. TRIM13, 24, and 29 , which are also involved in p53 regulation, have also been implicated as important regulators for carcinogenesis. Moreover, TRIM19/PML may act as a tumour suppressor protein (reviewed in [4]). TRIM22 expression is also downregulated in $\mathrm{CD} 4+\mathrm{T}$ cells from patients with active systemic lupus erythematosus (SLE) [59]. Although it is also unclear what role TRIM22 plays in this disease, it is notable that several other TRIM proteins, including TRIM 21, 25, 56, and 68 , have been linked to SLE and other autoimmune diseases [5]. It will be interesting to learn more about the role (if any) TRIM22 plays in these and other human diseases.

Although it is clear that TRIM22 is an exciting and dynamic protein, it appears that we have only begun to understand its role in cellular biology and antiviral immunity. A rich evolutionary history, together with its potential involvement in numerous biological processes, suggests that TRIM22 is an important and multifarious protein. Despite its importance, the function of TRIM22 remains poorly understood, and a number of issues will need to be addressed in future research. One discrepancy that needs clarification is the disparate observations and contradictory reports surrounding TRIM22 subcellular localization. In particular, we need to understand why TRIM22 localization is so heterogeneous, as this may provide useful insight into its biological function. Another priority will be to consolidate previous reports on the antiviral mechanism of TRIM22. In the case of HIV-1, it will be important to determine the stage(s) of the virus lifecycle that TRIM22 targets. In this regard, future studies that identify the host and/or virus targets of TRIM22 will be extremely useful. In addition, it will be interesting to discover if TRIM 22 has antiviral activity against additional viruses, and to determine the role it plays in other nonviral diseases. Overall, its breadth of involvement in antiviral immunity, combined with the range of possible mechanisms by which TRIM22 acts, presents a number of exciting research opportunities. Future work on TRIM22 will help us understand this important player in the host antiviral response and contribute to our knowledge of host-pathogen interactions.

\section{Acknowledgments}

S. D. Barr is supported by funds from the Department of Microbiology and Immunology (The University of Western Ontario) and a Scholarship Award from The Ontario HIV Treatment Network (OHTN). J. N. Kelly is supported by an Ontario Graduate Scholarship.

\section{References}

[1] K. Han, D. I. Lou, and S. L. Sawyer, "Identification of a genomic reservoir for new TRIM genes in primate genomes," PLoS Genetics, vol. 7, no. 12, Article ID e1002388, 2011.

[2] R. Rajsbaum, J. P. Stoye, and A. O'Garra, “Type I interferondependent and -independent expression of tripartite motif proteins in immune cells," European Journal of Immunology, vol. 38, no. 3, pp. 619-630, 2008.

[3] L. Carthagena, A. Bergamaschi, J. M. Luna et al., "Human TRIM gene expression in response to interferons," Plos one, vol. 4, no. 3, Article ID e4894, 2009. 
[4] S. Hatakeyama, "TRIM proteins and cancer," Nature Reviews Cancer, vol. 11, pp. 792-804, 2011.

[5] C. Jefferies, C. Wynne, and R. Higgs, "Antiviral TRIMs: friend or foe in autoimmune and autoinflammatory disease?" Nature Reviews Immunology, vol. 11, no. 9, pp. 617-625, 2011.

[6] L. M. van der Aa, J. P. Levraud, M. Yahmi et al., "A large new subset of TRIM genes highly diversified by duplication and positive selection in teleost fish," BMC Biology, vol. 7, article 7, 2009.

[7] S. L. Sawyer, M. Emerman, and H. S. Malik, "Discordant evolution of the adjacent antiretroviral genes TRIM22 and TRIM5 in mammals," PLoS Pathogens, vol. 3, no. 12, article e197, 2007.

[8] S. Nisole, J. P. Stoye, and A. Saïb, "TRIM family proteins: retroviral restriction and antiviral defence," Nature Reviews Microbiology, vol. 3, no. 10, pp. 799-808, 2005.

[9] G. Meroni and G. Diez-Roux, "TRIM/RBCC, a novel class of "single protein RING finger" E3 ubiquitin ligases," Bioessays, vol. 27, no. 11, pp. 1147-1157, 2005.

[10] Z. Duan, B. Gao, W. Xu, and S. Xiong, "Identification of TRIM22 as a RING finger E3 ubiquitin ligase," Biochemical and Biophysical Research Communications, vol. 374, no. 3, pp. 502-506, 2008.

[11] P. Eldin, L. Papon, A. Oteiza, E. Brocchi, T. G. Lawson, and N. Mechti, "TRIM22 E3 ubiquitin ligase activity is required to mediate antiviral activity against encephalomyocarditis virus," Journal of General Virology, vol. 90, no. 3, pp. 536-545, 2009.

[12] K. L. Lorick, J. P. Jensen, S. Fang, A. M. Ong, S. Hatakeyama, and A. M. Weissman, "RING fingers mediate ubiquitinconjugating enzyme (E2)-dependent ubiquitination," Proceedings of the National Academy of Sciences of the United States of America, vol. 96, no. 20, pp. 11364-11369, 1999.

[13] B. Gao, Z. Duan, W. Xu, and S. Xiong, "Tripartite motifcontaining 22 inhibits the activity of hepatitis b virus core promoter, which is dependent on nuclear-located RING domain," Hepatology, vol. 50, no. 2, pp. 424-433, 2009.

[14] S. D. Barr, J. R. Smiley, and F. D. Bushman, "The interferon response inhibits hiv particle production by induction of TRIM22," Plos Pathogens, vol. 4, no. 2, Article ID e1000007, 2008.

[15] M. Torok and L. D. Etkin, "Two B or not two B? overview of the rapidly expanding b-box family of proteins," Differentiation, vol. 67, no. 3, pp. 63-71, 2001.

[16] K. Ozato, D. M. Shin, T. H. Chang, and H. C. Morse, "TRIM family proteins and their emerging roles in innate immunity," Nature Reviews Immunology, vol. 8, no. 11, pp. 849-860, 2008.

[17] X. Li, D. F. Yeung, A. M. Fiegen, and J. Sodroski, "Determinants of the higher order association of the restriction factor TRIM $5 \alpha$ and other tripartite motif (TRIM) proteins," Journal of Biological Chemistry, vol. 286, no. 32, pp. 27959-27970, 2011.

[18] E. E. Nakayama and T. Shioda, "Anti-retroviral activity of TRIM5 $\alpha$," Reviews in Medical Virology, vol. 20, no. 2, pp. $77-$ 92, 2010.

[19] Z. Lukic and E. M. Campbell, "The cell biology of TRIM5 $\alpha$," Current HIV/AIDS Reports, vol. 9, pp. 73-80, 2012.

[20] G. Sivaramakrishnan, Y. Sun, R. Rajmohan, and V. C. L. Lin, "B30.2/SPRY domain in tripartite motif-containing 22 is essential for the formation of distinct nuclear bodies," FEBS Letters, vol. 583, no. 12, pp. 2093-2099, 2009.

[21] D. A. D. Parry, R. D. B. Fraser, and J. M. Squire, "Fifty years of coiled-coils and $\alpha$-helical bundles: a close relationship between sequence and structure," Journal of Structural Biology, vol. 163, no. 3, pp. 258-269, 2008.
[22] A. Reymond, G. Meroni, A. Fantozzi et al., "The tripartite motif family identifies cell compartments," EMBO Journal, vol. 20 , no. 9 , pp. 2140-2151, 2001.

[23] C. C. Mische, H. Javanbakht, B. Song et al., "Retroviral restriction factor TRIM $5 \alpha$ is a TRIMer," Journal of Virology, vol. 79, no. 22, pp. 14446-14450, 2005.

[24] A. Bouazzaoui, M. Kreutz, V. Eisert et al., "Stimulated transacting factor of $50 \mathrm{kDa}$ (Staf50) inhibits HIV-1 replication in human monocyte-derived macrophages," Virology, vol. 356, no. 1-2, pp. 79-94, 2006.

[25] A. Kajaste-Rudnitski, S. S. Marelli, C. Pultrone et al., "TRIM22 inhibits HIV-1 transcription independently of its E3 ubiquitin ligase activity, Tat, and NF- $\kappa$ B-responsive long terminal repeat elements," Journal of Virology, vol. 85, no. 10, pp. 5183-5196, 2011.

[26] R. Singh, G. Gaiha, L. Werner et al., "Association of TRIM22 with the type 1 interferon response and viral control duRING primary HIV-1 infection," Journal of Virology, vol. 85, no. 1, pp. 208-216, 2011.

[27] D. A. Rhodes, B. De Bono, and J. Trowsdale, "Relationship between spry and b30.2 protein domains. evolution of a component of immune defence," Immunology, vol. 116, no. 4, pp. 411-417, 2005.

[28] C. Grütter, C. Briand, G. Capitani et al., "Structure of the pryspry-domain: implications for autoinflammatory diseases," FEBS Letters, vol. 580, no. 1, pp. 99-106, 2006.

[29] S. Ohkura, M. W. Yap, T. Sheldon, and J. P. Stoye, "All three variable regions of the TRIM $5 \alpha$ B30.2 domain can contribute to the specificity of retrovirus restriction," Journal of Virology, vol. 80, no. 17, pp. 8554-8565, 2006.

[30] G. Sivaramakrishnan, Y. Sun, S. K. Tan, and V. C. L. Lin, "Dynamic localization of tripartite motif-containing 22 in nuclear and nucleolar bodies," Experimental Cell Research, vol. 315, no. 8, pp. 1521-1532, 2009.

[31] C. Tissot, S. A. Taviaux, S. Diriong, and N. Mechti, "Localization of Staf50, a member of the RING finger family, to $11 \mathrm{p} 15$ by ruorescence in situ hybridization," Genomics, vol. 34, no. 1, pp. 151-153, 1996.

[32] J. Zhang, S. Qin, S. N. J. Sait et al., "The pericentromeric region of human chromosome 11: evidence for a chromosomespecific duplication," Cytogenetics and Cell Genetics, vol. 94, no. 3-4, pp. 137-141, 2001.

[33] S. P. Otto and P. Yong, "The evolution of gene duplicates," Advances in Genetics, vol. 46, pp. 451-483, 2002.

[34] R. C. Moore and M. D. Purugganan, "The early stages of duplicate gene evolution," Proceedings of the National Academy of Sciences of the United States of America, vol. 100, no. 26, pp. 15682-15687, 2003.

[35] B. Conrad and S. E. Antonarakis, "Gene duplication: a drive for phenotypic diversity and cause of human disease," Annual Review of Genomics and Human Genetics, vol. 8, pp. 17-35, 2007.

[36] M. Sardiello, S. Cairo, B. Fontanella, A. Ballabio, and G. Meroni, "Genomic analysis of the TRIM family reveals two groups of genes with distinct evolutionary properties," BMC Evolutionary Biology, vol. 8, no. 1, article 225, 2008.

[37] S. L. Sawyer, L. I. Wu, M. Emerman, and H. S. Malik, "Positive selection of primate TRIM $5 \alpha$ identifies a critical species-specific retroviral restriction domain," Proceedings of the National Academy of Sciences of the United States of America, vol. 102, no. 8, pp. 2832-2837, 2005.

[38] M. W. Yap, S. Nisole, and J. P. Stoye, "A single amino acid change in the spry domain of human TRIM $5 \alpha$ leads to HIV-1 restriction," Current Biology, vol. 15, no. 1, pp. 73-78, 2005. 
[39] M. Stremlau, M. Perron, S. Welikala, and J. Sodroski, "Speciesspecific variation in the B30.2(SPRY) domain of TRIM5 $\alpha$ determines the potency of human immunodeficiency virus restriction," Journal of Virology, vol. 79, no. 5, pp. 3139-3145, 2005.

[40] A. M. Herr, R. Dressel, and L. Walter, "Different subcellular localisations of TRIM22 suggest species-specific function," Immunogenetics, vol. 61, no. 4, pp. 271-280, 2009.

[41] S. Yu, B. Gao, Z. Duan, W. Xu, and S. Xiong, "Identification of tripartite motif-containing 22 (TRIM22) as a novel NF- $\kappa \mathrm{B}$ activator," Biochemical and Biophysical Research Communications, vol. 410, no. 2, pp. 247-251, 2011.

[42] J. Petersson, P. Lönnbro, A. M. Herr, M. Mörgelin, U. Gullberg, and K. Drott, "The human IFN-inducible p53 target gene TRIM22 colocalizes with the centrosome independently of cell cycle phase," Experimental Cell Research, vol. 316, no. 4, pp. 568-579, 2010.

[43] X. Li, B. Gold, C. O’hUigin et al., "Unique features of TRIM $5 \alpha$ among closely related human TRIM family members," Virology, vol. 360, no. 2, pp. 419-433, 2007.

[44] M. Cioce and A. I. Lamond, "Cajal bodies: a long history of discovery," Annual Review of Cell and Developmental Biology, vol. 21, pp. 105-131, 2005.

[45] C. Tissot and N. Mechti, "Molecular cloning of a new interferon-induced factor that represses human immunodeficiency virus type i long terminal repeat expression," Journal of Biological Chemistry, vol. 270, no. 25, pp. 14891-14898, 1995.

[46] S. Obad, H. Brunnström, J. Vallon-Christersson, A. Borg, K. Drott, and U. Gullberg, "Staf50 is a novel p53 target gene conferRING reduced clonogenic growth of leukemic U-937 cells," Oncogene, vol. 23, no. 23, pp. 4050-4059, 2004.

[47] C. Gongora, C. Tissot, C. Cerdan, and N. Mechti, "The interferon-inducible Staf50 gene is downregulated during t cell costimulation by CD2 and CD28," Journal of Interferon and Cytokine Research, vol. 20, no. 11, pp. 955-961, 2000.

[48] B. Gao, Y. Wang, W. Xu, Z. Duan, and S. Xiong, "A 55' extended IFN-stimulating response element is crucial for IFN$\gamma$-induced tripartite motif 22 expression via interaction with ifn regulatory factor-1," Journal of Immunology, vol. 185, no. 4, pp. 2314-2323, 2010.

[49] Y. Wang, B. Gao, W. Xu, and S. Xiong, "Brg1 is indispensable for IFN- $\gamma$-induced TRIM22 expression, which is dependent on the recruitment of IRF-1," Biochemical and Biophysical Research Communications, vol. 410, no. 3, pp. 549-554, 2011.

[50] S. Obad, T. Olofsson, N. Mechti, U. Gullberg, and K. Drott, "Regulation of the interferon-inducible p53 target gene TRIM22 (Staf50) in human t lymphocyte activation," Journal of Interferon and Cytokine Research, vol. 27, no. 10, pp. 857864, 2007.

[51] S. Wieland, R. Thimme, R. H. Purcell, and F. V. Chisari, "Genomic analysis of the host response to hepatitis b virus infection," Proceedings of the National Academy of Sciences of the United States of America, vol. 101, no. 17, pp. 6669-6674, 2004.

[52] A. I. Su, J. P. Pezacki, L. Wodicka et al., "Genomic analysis of the host response to hepatitis C virus infection," Proceedings of the National Academy of Sciences of the United States of America, vol. 99, no. 24, pp. 15669-15674, 2002.

[53] M. E. Folkers, D. A. Delker, C. I. Maxwell et al., "Encode tiling array analysis identifies differentially expressed annotated and novel 5' capped RNAs in hepatitis C infected liver," Plos ONE, vol. 6, no. 2, Article ID e14697, 2011.

[54] X. Y. Mo, W. Ma, Y. Zhang et al., "Microarray analyses of differentially expressed human genes and biological processes in ECV304 cells infected with rubella virus," Journal of Medical Virology, vol. 79, no. 11, pp. 1783-1791, 2007.

[55] E. Izmailova, F. M. N. Bertley, Q. Huang et al., "HIV-1 Tat reprograms immature dendritic cells to express chemoattractants for activated T cells and macrophages," Nature Medicine, vol. 9, no. 2, pp. 191-197, 2003.

[56] Y. E. Chang and L. A. Laimins, "Microarray analysis identifies interferon-inducible genes and Stat-1 as major transcriptional targets of human papillomavirus type 31," Journal of Virology, vol. 74, no. 9, pp. 4174-4182, 2000.

[57] Y. Wang, H. Li, Q. Tang, G. G. Maul, and Y. Yuan, "Kaposi's sarcoma-associated herpesvirus ori-lyt-dependent DNA replication: involvement of host cellular factors," Journal of Virology, vol. 82, no. 6, pp. 2867-2882, 2008.

[58] O. Bandman, R. T. Coleman, J. F. LoRING, J. J. Seilhamer, and B. G. Cocks, "Complexity of inflammatory responses in endothelial cells and vascular smooth muscle cells determined by microarray analysis," Annals of the New York Academy of Sciences, vol. 975, pp. 77-90, 2002.

[59] Y. J. Deng, Z. X. Huang, C. J. Zhou et al., "Gene profiling involved in immature $\mathrm{CD}^{+}{ }^{+} \mathrm{T}$ lymphocyte responsible for systemic lupus erythematosus," Molecular Immunology, vol. 43, no. 9, pp. 1497-1507, 2006.

[60] B. Zirn, O. Hartmann, B. Samans et al., "Expression profiling of wilms tumors reveals new candidate genes for different clinical parameters," International Journal of Cancer, vol. 118, no. 8, pp. 1954-1962, 2006.

[61] S. Wittmann, C. Wunder, B. Zirn et al., "New prognostic markers revealed by evaluation of genes correlated with clinical parameters in wilms tumors," Genes Chromosomes and Cancer, vol. 47, no. 5, pp. 386-395, 2008.

[62] T. X. Liu, J. W. Zhang, J. Tao et al., "Gene expression networks underlying retinoic acid-induced differentiation of acute promyelocytic leukemia cells," Blood, vol. 96, no. 4, pp. 1496-1504, 2000.

[63] S. Obad, T. Olofsson, N. Mechti, U. Gullberg, and K. Drott, "Expression of the IFN-inducible p53-target gene TRIM22 is down-regulated duRING erythroid differentiation of human bone marrow," Leukemia Research, vol. 31, no. 7, pp. 9951001, 2007. 

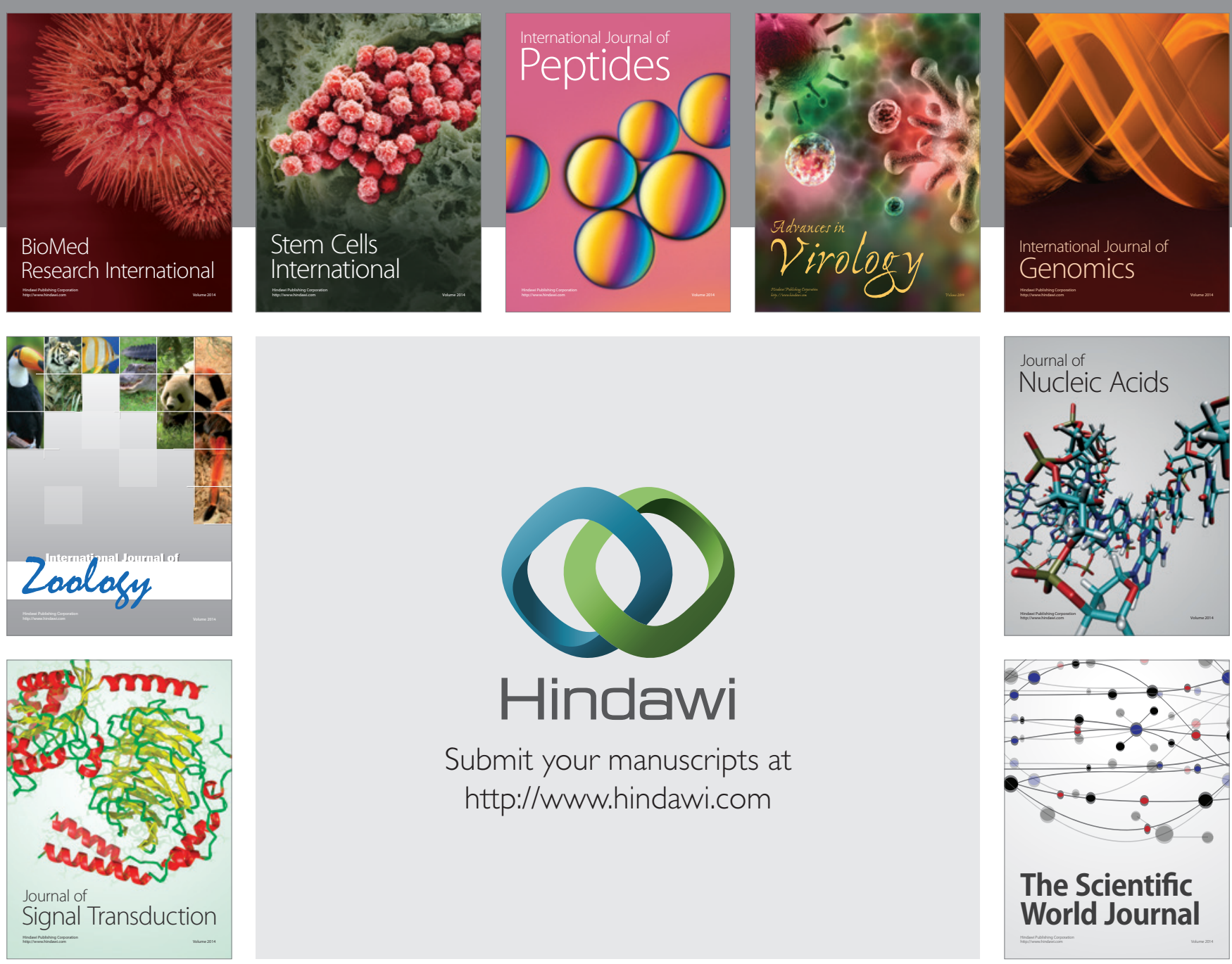

Submit your manuscripts at

http://www.hindawi.com
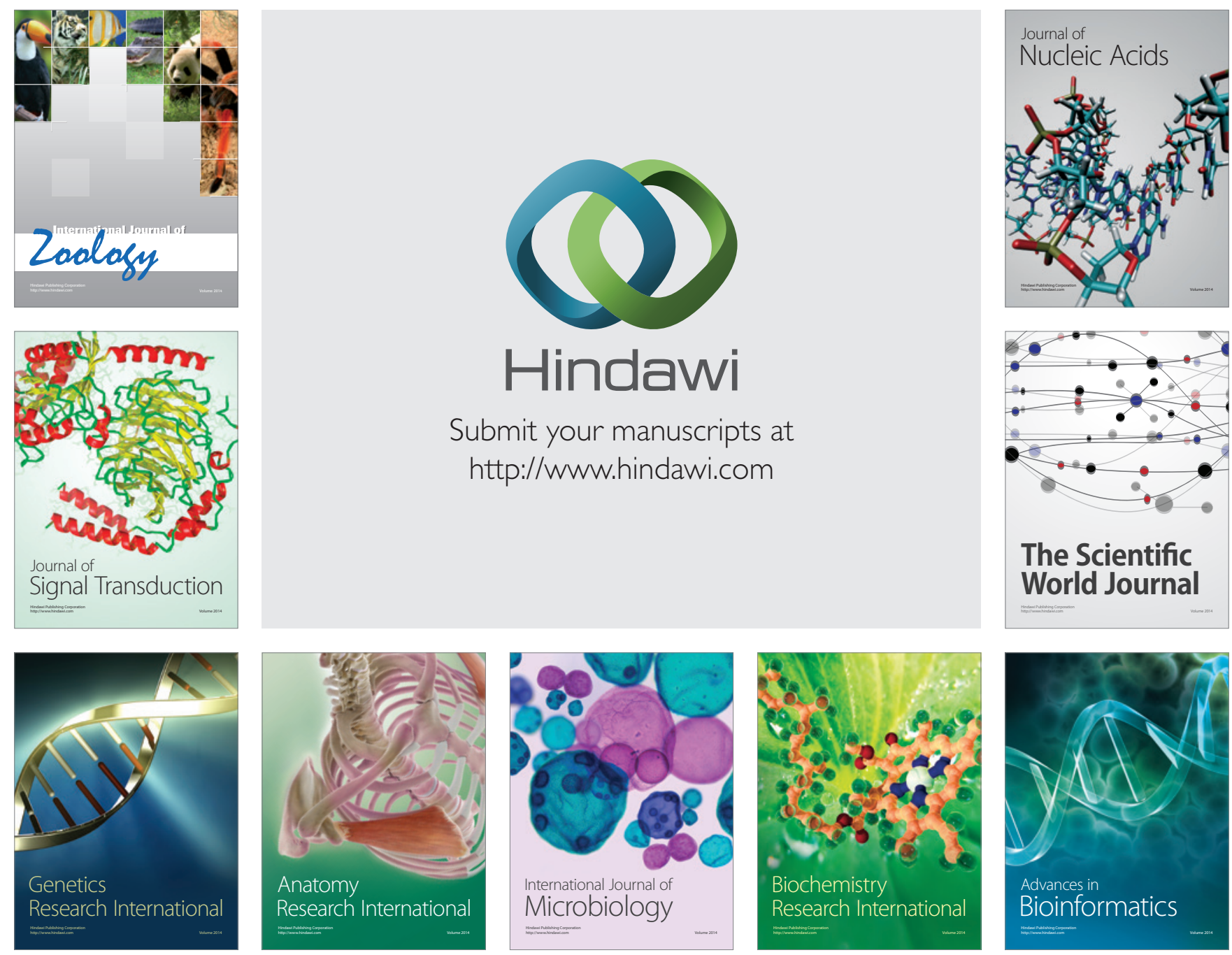

The Scientific World Journal
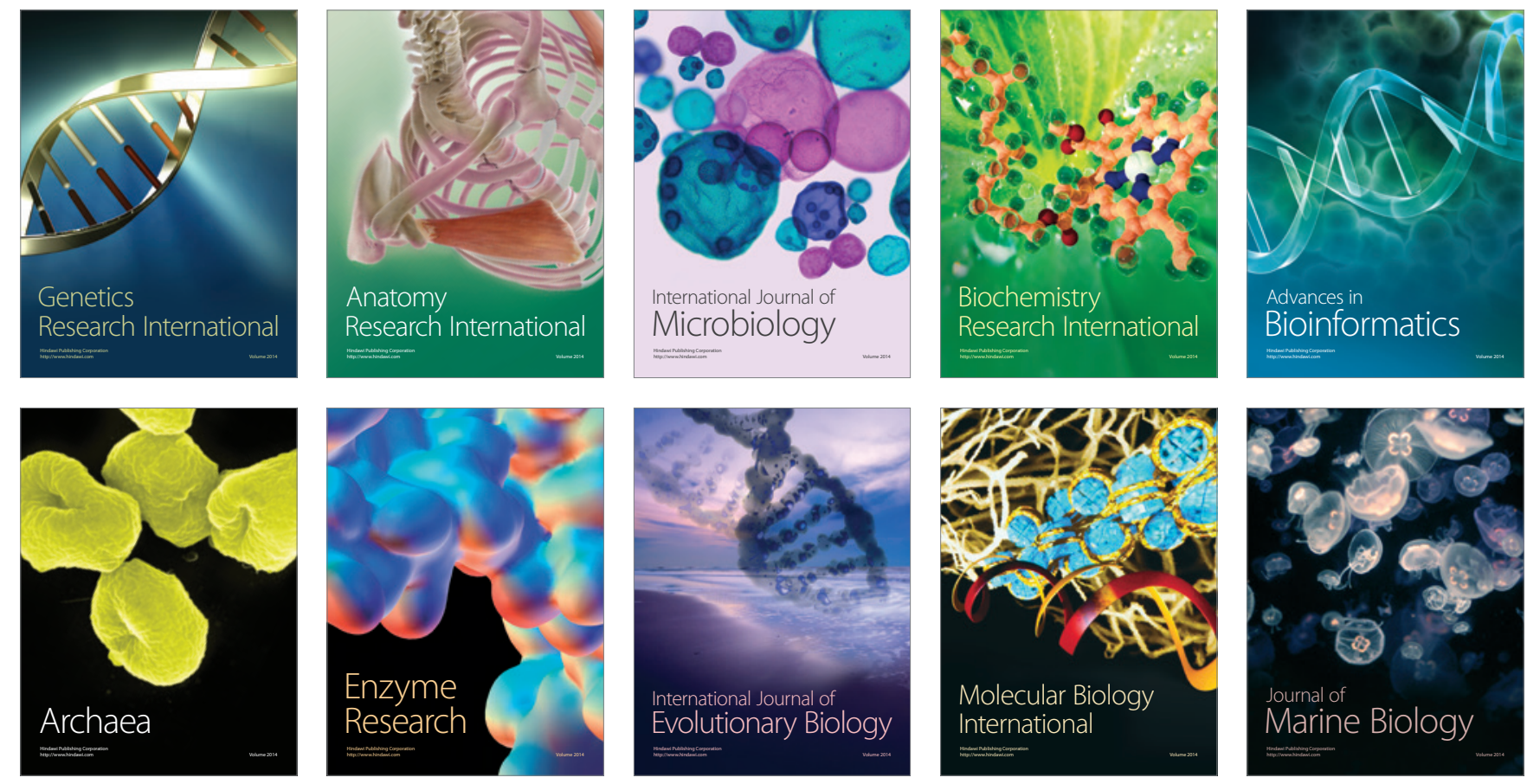